\title{
Robotic Percutaneous Coronary Intervention: The Good, the Bad, and What is to Come
}

Laura Young (1) and Jaikirshan Khatri (1)

Heart, Vascular \& Thoracic Institute, Cleveland Clinic, Cleveland, $\mathrm{OH}$

\begin{abstract}
The introduction of robots into healthcare has brought a wealth of opportunity for technical advancements, ranging from cleaning robots to disinfect hospital rooms to the high-tech surgical robots used in the operating room. Robotic-assisted percutaneous coronary intervention (R-PCI) has been a more recent development in the field, and is particularly revolutionary in that it serves to benefit the interventional cardiologist as well as the patient. Published data on R-PCI have shown its feasibility, safety, and more recently, its potential benefits. This review examines the current role of the robot in the catheterization laboratory, the authors' experience with the most current generation of the robot, and what is yet to come.
\end{abstract}

\section{Keywords}

Robotics, percutaneous coronary intervention, complex percutaneous coronary intervention, interventional devices, innovation

\section{Disclosure: The authors have no conflicts of interest to declare.}

Received: November 22, 2020 Accepted: September 6, 2021 Citation: US Cardiology Review 2022;16:e02. DOI: https://doi.org/10.15420/usc.2020.28R1 Correspondence: Laura Young, 9500 Euclid Ave, J2-3, Cleveland, OH 44195. E: youngL8@ccf.org

Open Access: This work is open access under the CC-BY-NC 4.0 License which allows users to copy, redistribute and make derivative works for non-commercial purposes, provided the original work is cited correctly.

The field of percutaneous coronary intervention (PCI) has rapidly evolved since its inauguration in 1977. Its constant adaptation and advancement have allowed it to remain a mainstay in the treatment of patients with coronary artery disease and acute coronary syndromes. Logically, the majority of technological advancements within the field have focused on ensuring patient safety and expanding the scope of which transcatheter interventions can be used. While there have been significant innovations in what we can do, adaptations in how we perform $\mathrm{PCl}$ have lagged behind. As a result, operator fatigue and occupational hazards related to both radiation exposure and orthopedic injury from prolonged standing remain top concerns of interventional cardiologists. Robotic-assisted $\mathrm{PCl}$ $(\mathrm{R}-\mathrm{PCl})$ is one of the novel innovations within interventional cardiology, aiming to address both occupational hazards for the operator alongside procedural quality and safety improvement for the patient. We review the current role of the robot in the catheterization laboratory, the current systems available for use, and our experience with R-PCI.

\section{The Potential of Robotics in the Catheterization Laboratory}

When initially conceptualized, the appeal of a robotic system in the catheterization laboratory reflected that seen in the operating room, where the robot could provide a means of standardizing procedural precision and reproducibility while also addressing occupational hazards of the primary operator. The concept was to create a remote system that would allow for the primary operator to sit behind a shielded console, colloquially termed the 'cockpit', thereby reducing radiation exposure and orthopedic injury from prolonged standing at the bedside in a full lead apron. There has been widespread recognition of the risks of long-term radiation exposure, with a definitively increased risk of cataracts and numerous malignancies involving the thyroid, brain, and bone marrow. ${ }^{1-3}$ Lead shielding has remained the mainstay of radiation protection for staff throughout the decades. While its armamentarium has expanded from lead aprons to include more novel ideas, such as lead-lined gloves and hats, and 'zero-gravity' lead (ceiling-suspended lead aprons), the overall paradigm remains unchanged, focusing on personal protective equipment. The incidence of orthopedic injury amongst interventional cardiologists remains alarmingly high, with $49 \%$ of interventionalists reporting at least one orthopedic injury in a 2014 nationwide survey. Robotic-assisted $\mathrm{PCl}$ offers a novel modality that can combat both radiation exposure and orthopedic injury for the interventionalist.

The robotic system also provides a potential for precision of device delivery that could supersede what can be achieved by the human eye, particularly when standing a foot away from the screen. The ability to control the robotic arm to move equipment sub-millimeter amounts with such accuracy would certainly be beneficial in the interventional cardiology realm, where accurate stent and balloon placement is critical. Furthermore, the algorithmic capability of a robotic system could create the potential for standardization of the procedure by equalizing the skill set of operators in guidewire navigation and device delivery.

Removing the primary operator from the bedside opens the possibility of remote procedures, a concept that has colloquially been termed 'telestenting'. The idea of remote operator PCl seemed futuristic a decade ago, but Patel et al. proved its feasibility in early 2019. ${ }^{6}$ They had a primary operator perform robotic $\mathrm{PCl}$ for five patients with type $\mathrm{A}$ coronary lesions while situated 20 miles away from the catheterization laboratory. The operator was able to perform the robotic PCl successfully 
Figure 1: Robotic Percutaneous Coronary Intervention in the Cleveland Clinic Catheterization Laboratory

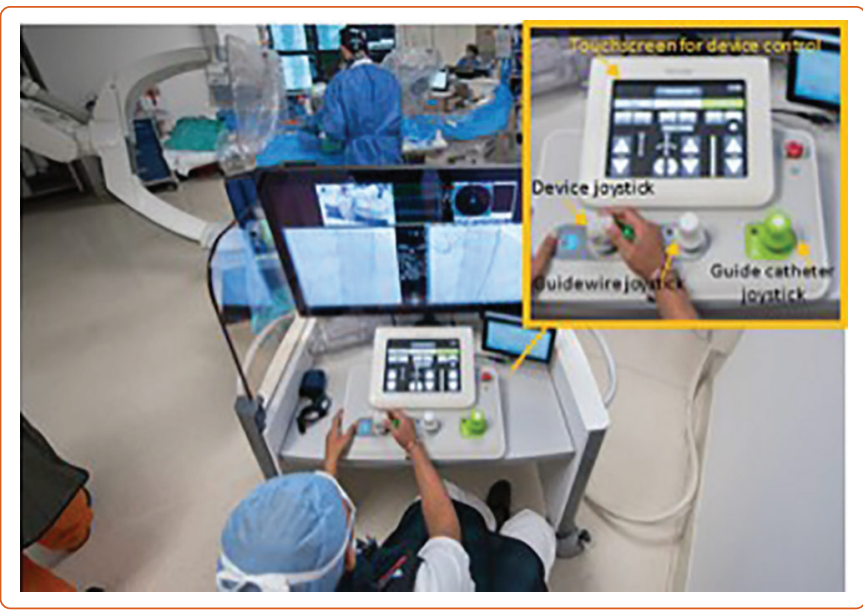

The general setup of our robotic percutaneous coronary intervention cases at the Cleveland Clinic. One of the interventional cardiology operators sits in the cockpit to control the robotic arm, while the other is at bedside to assist with device exchanges and manipulation of the image intensifier.

\section{Figure 2: Anatomy of the Robotic Single-use Cassette}

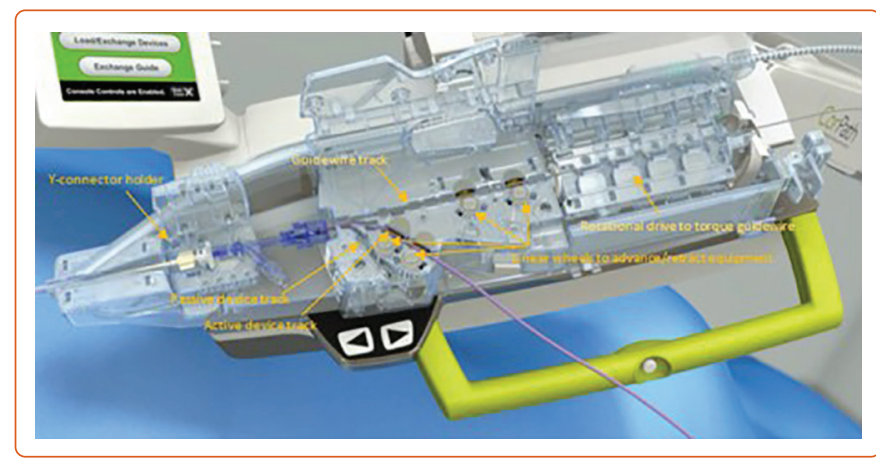

Close up view of the CorPath GRX robotic arm, mounted with the single use cassette

without conversion to a manual approach in all of the patients. In early 2020, Madder et al. demonstrated the feasibility of transcontinental telestenting in preclinical models, where the operator was located over 3,000 miles away from the ex vivo model. ${ }^{7}$ They found that there was no difference in performance and safety when telestenting was performed regionally versus transcontinental. As would be expected, they found that there was greater latency of media transmission in the transcontinental cases, although this was qualified as imperceptible by the operators. Telestenting is still in its infancy but ultimately could serve a multitude of purposes. Its biggest appeal has been to improve $\mathrm{PCl}$ access in remote and underserved regions. It could at the very least allow experts to aid in complex procedures from afar, widening the reach of these highly skilled operators. Furthermore, in the era of the COVID-19 pandemic, we must consider its utility in treating highly infectious patients while keeping our staff safe.

\section{Systems}

There are currently two commercially available robotic systems for $\mathrm{PCl}$ : the CorPath (Corindus) and the R-One (Robocath). The CorPath 200 was the first $\mathrm{PCl}$ robotic system to receive Food and Drug Administration clearance in 2005 and Corindus has since introduced their secondgeneration system, CorPath GRX. The R-One system by Robocath received a CE marking in February 2019 and is currently available throughout Europe and Africa. It has similar features to the first iteration of the
Corindus Vascular System, the CorPath 200. While it currently lags in technical advancements, the inherent rivalry between systems is encouraging to promote innovation within the field.

The systems are made up of two subunits - the robotic arm that is stationed bedside and the interventional cockpit, where the primary operator will sit to perform the $\mathrm{PCl}$ (Figures 1 and 2). The robotic arm is stationed at the caudal end of the table and can be retracted to the side when not in use. A single use cassette is attached to the arm in a sterile fashion and contains three equipment tracks: one designated for the guidewire, an active track for device advancement using the robot, and a passive track for devices that are left in place (such as a guide catheter extension) or that are used in a hybrid approach with manual advancement of the device by the bedside operator.

The interventional cockpit can be placed in the catheterization laboratory behind a radiation shield or alternatively can be placed in the control room. Either way, the primary operator is able to perform the $\mathrm{PCl}$ without the orthopedic burden of a lead apron. The setup of the cockpit varies amongst the different systems, but the general concept is similar. There is a high-resolution screen that allows the operator to monitor the fluoroscopy screen, hemodynamics, and the bedside operations simultaneously (the latter from a camera that is setup above the table). The gears of the robot are then controlled by individual joysticks, which manipulate the equipment in an assigned track. Both systems have a joystick for the guidewire and one for the device, while the CorPath GRX has a third joystick for control of the guide catheter.

\section{Data}

A number of observational studies have been published evaluating the feasibility, safety, and potential benefits of R-PCl for both patients and operators. Given the relatively recent approval of the Robocath R-One for commercial use, the majority of data have come from use of the Corindus CorPath robot models. The preclinical randomized trial for the R-One device was conducted by three operators in 2017 , with a $100 \%$ technical success rate and no major adverse cardiac events. ${ }^{8}$ The data overall have been positive in terms of safety, feasibility, and short-term outcomes. A recent meta-analysis reflects this, including $148 \mathrm{R}-\mathrm{PCl}$ patients from five studies in comparison to 493 patients who underwent manual PCl. They found that operators had lower radiation exposure in the R-PCl group without compromise in total stents per case, fluoroscopy time, or procedural success rates. $^{9}$

The PRECISE study was the inaugural study to evaluate safety and feasibility of robotic $\mathrm{PCl}$, published by Weisz et al. in 2013.10 This nonrandomized, multicenter study enrolled patients with at least 50\% coronary stenosis that could be treated with a single stent, with the majority of lesions being classified as type A (28.7\%) or B1 (39.6\%). These stringent inclusion criteria essentially allowed this study to serve as a proof of concept for $\mathrm{R}-\mathrm{PCl}$. The results were favorable, with technical success achieved in 162 of 164 patients (98.8\%) without conversion to manual operation. At 30-day follow-up, there were no deaths, strokes, non-fatal MI, or target lesion revascularization. Perhaps the most exciting result though was the significant reduction in median radiation exposure of the primary operator, which was found to be $95.2 \%$ less during time spent in the interventional cockpit compared to time spent at the traditional table position.

CORA-PCl was designed to assess the feasibility of R-PCl for more complex patient lesions, whereby the inherently longer procedural 
catheter has been engaged should it be connected to the robotic arm. The guide catheter is locked into place within the cassette, which inhibits further manual adjustments unless the robotic arm is disconnected. The CorPath GRX system has an upgraded feature in attempt to allow for robotic guide catheter control through joystick manipulation. This is certainly a vast improvement from the prior iteration, there was no alternative to disconnecting the system and reengaging the coronary manually. While ideal for subtle adjustments of the guide catheter during the case, we have found it very difficult to reengage the guide catheter entirely using the robot alone despite anecdotal reports of this being possible. Instead, we find it much more efficient to briefly disconnect the guide catheter for manual manipulation. The joystick allows for advancement and retraction as well as torquing of the guide catheter. It should be noted that advancement and retraction will result in movement of all connected devices, and so guidewires and catheters should be watched closely to prevent inadvertent complications. We have also learned that $90 \mathrm{~cm}$ guide catheters, often used in CTO cases for retrograde access, are often too short to complete $\mathrm{PCl}$ robotically. When connected to the robotic arm, these shorter guide catheters create too much tension to maintain stable coronary engagement. In taller patients, $90 \mathrm{~cm}$ guide catheters lack the necessary length to connect to the robotic cassette. With the first generation of the CorPath robotic system, aorto-ostial lesions were difficult due to the inability to control the guide catheter. Now with the upgraded system, ostial lesions are feasible as long as the operator has adequate ability to actively control the guide catheter. If the patient has tortuous anatomy or radial spasm, an alternative approach should be considered.

Similar to guide catheter manipulation with the robot, robotic guidewire navigation requires the operator to rely only on visual feedback. The robot can accommodate any 0.014 inch guidewire within its track. It should be noted that the current robotic iterations do not support the use of pressure guidewires for invasive hemodynamics, though the guidewire can be used once disconnected from the modular connector. One of the major appeals of $\mathrm{R}-\mathrm{PCl}$ is the computational capability of the robot, which could allow for the creation of algorithms based on techniques of advanced operators - essentially leveling the playing field for all interventionalists. Corindus recently released its upgraded software for the CorPath GRX, including a proprietary program called 'Rotate on Retract', which is the first installation of its proprietary technlQ Smart Procedural Automation. The automation was designed to improve procedural reproducibility independent of an operator's individual skills by creating algorithms based on the experience of highly skilled interventional cardiologists. When the 'Rotate on Retract' feature is activated, the robot will automatically rotate the guidewire $270^{\circ}$ with each retraction input, so as to set the operator up for an alternative approach as one would at the bedside. Objective, preclinical data were favorable when the feature was used for wiring coronaries in a porcine model. ${ }^{15}$ It is also possible to torque the guidewire manually using the joystick, although there is a significant delay between input and wire response. We look forward to the implementation of future automated movements including spin, wiggle, Dotter, and constant speed, all of which could greatly improve robotic navigation and lesion crossing.

When considering device delivery, the robot is impartial in terms of device companies and can accommodate rapid exchange catheters up to $7 \mathrm{Fr}$ (although off label, we have been able to use 8 Fr guide catheters without issue). Over-the-wire equipment, such as rotational or orbital atherectomy systems, cannot yet be accommodated for R-PCl. While it is possible to perform atherectomy and then transition to a robotic approach, this cannot be done with a hybrid approach. Microcatheters, a fundamental tool for $\mathrm{PCl}$ of chronic total occlusions, cannot be accommodated either, which makes attempts at robotic wiring of these lesions implausible with the current systems. The gears within the system also prohibit the use of fragile catheters that lack a rigid hypotube, such as the Beta-Cath for brachytherapy (Novoste) or the Dragonfly Optis catheter for optical coherence tomography imaging (Abbott).

Intravascular imaging is a crucial step in our daily $\mathrm{PCl}$ practice so it is imperative that we can incorporate it into the workflow of R-PCl. The robotic systems cannot accommodate automatic pullback runs of rotational devices, although this is not an issue in our laboratory as we predominantly use solid-state intravascular ultrasound (IVUS) imaging. While it is possible to perform a pullback robotically using the device joystick, we have found that the robotic gears can occasionally damage the IVUS catheter and render it defective. This often occurs in calcified or tortuous lesions, where the robotic gears slip as the IVUS catheter is gripped within the vessel. As a large majority of our patients have at least one of these two vessel characteristics, we prefer to use IVUS in a hybrid approach by placing the catheter in the passive device track, which allows the catheter to be advanced and retracted manually by the bedside operator.

One of the major benefits that we have found in using the $\mathrm{R}-\mathrm{PCl}$ relates to its ability to assist in precision with measurements. The CorPath GRX has patented software that allows for lesion length measurement during pullback of any intracoronary device. The distal marker or end of the device is placed at the distal target lesion border and the device position counter is then reset to zero. The operator can then retract the device until the distal marker is at the proximal target lesion border, where the total length traveled will be displayed on screen in mm. Appropriate stent length, even when multiple stents are needed, can then be chosen without any guesswork. It should be noted that accuracy of the software depends on 1:1 joystick input to movement responsiveness of the device, which is not always the case. If the device is stuck, the program will still count and render an inaccurate measurement. We find that this often happens with the IVUS catheter but is less of an issue with balloons or stents. Prior data have also suggested that R-PCl lowers the incidence of longitudinal geographic miss compared to manual PCl. ${ }^{16}$ This is likely related to a combination of improved measurement accuracy with the robotic software, improved visualization given closer screen proximity for the operator, and finally the controlled pinning of the device during inflation and deployment by the robot gears.

While we have found it to be relatively uncommon to need to abort a robotic approach, conversion to manual $\mathrm{PCl}$ is quite straightforward. The guide catheter must be unlocked from the robotic arm, which thereby frees up the catheter and equipment for manual use and the robot can then be positioned at the end of the bed. This step is not particularly cumbersome but does add about a minute of procedural time. On our experience, we have not found this to be detrimental to our workflow.

Overall, we have found that $\mathrm{R}-\mathrm{PCl}$ can be easily incorporated into daily use for non-complex coronary lesions and is certainly feasible for complex coronary lesions. Catheterization laboratories do have to be thoughtful about resource utilization and financial burden of novel equipment. Encouragingly, data are starting to emerge suggesting that the cost of $\mathrm{R}-\mathrm{PCl}$ is comparable to manual cases overall, with the expected increase in direct supplies cost related to the single-use robotic equipment. ${ }^{16,17}$ At our institution, we see this cost can be seen as a long-term investment for our operators' radiation safety and long-term health with reduced 
orthopedic burden from standing in lead. Economically, R-PCl has the potential to improve our accuracy leading to cost savings in terms of percase stent use; preliminary data have suggested that this holds true, though dedicated studies are needed to validate this potential. ${ }^{17}$

\section{Conclusion}

There is no doubt that R-PCl has significant potential for expanding our capabilities in the catheterization laboratory, whether regarding standardization of the procedure, improved operator endurance, or the prospect of telestenting. While we have made significant strides in the technical capabilities of R-PCl over the past decade, robotic systems will need to continue to evolve to adapt for the needs of the procedure. With the influx of data supporting intravascular imaging for optimization of $\mathrm{PCl}$, it is imperative that upgrades be made so that these devices can seamlessly and reliably be used within the robotic system. Likewise, the routine need for over-the-wire equipment in complex $\mathrm{PCl}$ cases must be addressed. While there is still a way to go, it is important to recognize how far we have come. We must continue to purposefully practice the workflow of R-PCl to improve our efficiency and unleash the potential of this technology. $\square$
1. Roguin A, Goldstein J, Bar O, Goldstein JA. Brain and neck tumors among physicians performing interventional procedures. Am J Cardiol 2013;111:1368-72. https://doi. org/10.1016/j.amjcard.2012.12.060; PMID: 23419190.

2. Zielinski JM, Garner MJ, Band PR, et al. Health outcomes of low-dose ionizing radiation exposure among medical workers: a cohort study of the Canadian national dose registry of radiation workers. Int J Occup Med Environ Health 2009;22:149-56. https://doi.org/10.2478/v10001-0090010-y; PMID: 19546093

3. Karatasakis A, Brilakis HS, Danek BA, et al. Radiationassociated lens changes in the cardiac catheterization laboratory: Results from the IC-CATARACT (CATaracts Attributed to RAdiation in the CaTh lab) study. Catheter Cardiovasc Interv 2018;91:647-54. https://doi.org/10.1002 ccd.27173; PMID: 28707381

4. Andreassi MG, Piccaluga E, Guagliumi G, et al. Occupationa health risks in cardiac catheterization laboratory workers. Circ Cardiovasc Interv 2016;9:e003273. https://doi.org/10.1161/ CIRCINTERVENTIONS.115.003273; PMID: 27072525.

5. Klein LW, Tra Y, Garratt KN, et al. Occupational health hazards of interventional cardiologists in the current decade: results of the 2014 SCAl membership survey. Catheter Cardiovasc Interv 2015;86:913-24. https://doi. org/10.1002/ccd.25927; PMID: 25810341.

6. Patel TM, Shah SC, Pancholy SB. Long distance tele-robotic assisted percutaneous coronary intervention: a report of first-in-human experience. EClinicalMedicine 2019;14:53-8. https://doi.org/10.1016/j.eclinm.2019.07.017; PMID: 31709402

7. Madder RD, VanOosterhout S, Parker J, et al. Robotic telestenting performance in transcontinental and regional pre-clinical models. Catheter Cardiovasc Interv 2021;97:E32732. https://doi.org/10.1002/ccd.29115; PMID: 32583944.

8. Robocath Demonstrates Safety and Efficacy OF R-One. Robocath Robocath Demonstrates Safety and Efficacy of ROne Comments. https://www.robocath.com/robocathdemonstrates-safety-and-efficacy-of-r-one/ (accessed 14 December 2021).

9. Allencherril J, Hyman D, Loya A, et al. Outcomes of robotically assisted versus manual percutaneous coronary intervention: a systematic review and meta-analysis. J Invasive Cardiol 2019;31:199-203; PMID: 31088991.

10. Weisz G, Metzger DC, Caputo RP, et al. Safety and feasibility of robotic percutaneous coronary intervention: PRECISE (Percutaneous Robotically-Enhanced Coronary Intervention) study. J Am Coll Cardiol 2013;61:1596-600. https://doi. org/10.1016/j.jacc.2012.12.045; PMID: 23500318.

11. Mahmud E, Naghi J, Ang L, et al. Demonstration of the safety and feasibility of robotically assisted percutaneous coronary intervention in complex coronary lesions: Results of the CORA-PCl study (Complex Robotically Assisted Percutaneous Coronary Intervention). JACC Cardiovasc Inter 2017;10:1320-7. https://doi.org/10.1016/j.jcin.2017.03.050; PMID: 28683937

12. Hirai T, Kearney K, Kataruka A, et al. Initial report of safety and procedure duration of robotic-assisted chronic total occlusion coronary intervention. Catheter Cardiovasc Interv 2020;95:165-9. https://doi.org/10.1002/ccd.28477; PMID: 31483078.

13. Patel TM, Shah SC, Soni YY, et al. Comparison of robotic percutaneous coronary intervention with traditional percutaneous coronary intervention: A propensity scorematched analysis of a large cohort. Circ Cardiovasc Interv 2020;13:e008888. https://doi.org/10.1161/ CIRCINTERVENTIONS.119.008888; PMID: 32406263.

14. Nagaraja V, Khatri JJ. Hybrid robotic impella assisted single arterial access complex high risk percutaneous coronary intervention. Cardiovasc Revasc Med 2020;21:105-7. https:/ doi.org/10.1016/j.carrev.2019.12.007; PMID: 31948848.

15. Madder R LW, Parikh M, Kandzari D, et al. TCT-539. Impact of a novel advanced robotic wiring algorithm on time to wire a coronary artery bifurcation in a porcine model. J Am Coll Cardiol 2017;70(18 Suppl):B223. https://doi.org/10.1016/. jacc.2017.09.712.

16. Bezerra HG, Mehanna E, Vetrovec GW, et al. Longitudinal geographic miss (IGM) in robotic assisted versus manual percutaneous coronary interventions. J Interv Cardiol 2015;28:449-55. https://doi.org/10.1111/joic.12231; PMID: 26489972

17. Hamandi M, Cobb R, Foster L, et al. Cost and efficacy analysis of robotically assisted percutaneous coronary interventions. J Am Coll Cardio/ 2019;73(9 Suppl 1):1114. https://doi.org/10.1016/S0735-1097(19)31721-8. 